Please do not remove this page

RMIT

UNIVERSITY

\title{
Refining As-cast beta-Ti Grains Through ZrN Inoculation
}

Qiu, Dong; Zhang, Duyao; Easton, Mark; StJohn, David; Gibson, Mark

https://researchrepository.rmit.edu.au/esploro/outputs/9921863274901341/filesAndLinks?institution=61RMIT_INST\&index=null

Qiu, D., Zhang, D., Easton, M., StJohn, D., \& Gibson, M. (2018). Refining As-cast beta-Ti Grains Through ZrN Inoculation. Metallurgical and Materials Transactions A: Physical Metallurgy and Materials Science, 49(5), 1444-1449. https://doi.org/10.1007/s11661-018-4531-5

Document Version: Accepted Manuscript

Published Version: https://doi.org/10.1007/s11661-018-4531-5

Repository homepage: https://researchrepository.rmit.edu.au

(c) The Minerals, Metals \& Materials Society and ASM International 2018

Downloaded On 2023/04/26 20:23:28 +1000

Please do not remove this page 
$4{ }^{a}$ School of Engineering, RMIT University, Carlton, Victoria, Australia

${ }^{\mathrm{b}}$ School of Mechanical and Mining Engineering, University of Queensland, St Lucia, QLD,

6 Australia

${ }^{\mathrm{C}}$ CSIRO Manufacturing, Clayton, Victoria, Australia

* Corresponding author. Tel: +61-3-9925-4487. Email address: dong.qiu2@rmit.edu.au

Abstract

The columnar-to-equiaxed transition and remarkable refinement of $\beta$-Ti grains occurs in an as-cast Ti-13Mo alloy when a new grain refiner, $\mathrm{ZrN}$, was inoculated at a nitrogen level as low as 0.4 wt. \%. The grain refining effect is attributed to in-situ formed TiN particles that provide active nucleation sites and solute $\mathrm{Zr}$ that promotes constitutional supercooling.

Reproducible orientation relationships were identified between TiN nucleants and $\beta$-Ti matrix, and well explained by the edge-to-edge matching model.

Keywords: grain refinement; titanium alloy; heterogeneous nucleation; inoculation

It is acknowledged that grain refinement of engineering alloys during solidification can significantly reduce casting defects, enhance mechanical properties and improve formability during subsequent thermomechanical processing [1]. In addition to the benefit to conventional cast and forged products, fine and equiaxed grains also play a critical role for 
additively manufactured alloy components. Smart manipulation of the material's chemistry and laser/electron beam scanning strategies can convert commonly observed columnar grains, fully or partially, into equiaxed grains, reducing the microstructural anisotropy and suppressing the formation of hot cracks in as-built components [2-4].

Unlike other widely used engineering alloys, such as Mg alloys, Al alloys and steels, there is currently no commercial grain refiner available for Ti and its alloys. So far, the best grain refining efficiency was obtained through the additions of boron as a solute due to its strong segregating power $[5,6]$. However, in the absence of potent inoculant particles, trace boron addition as solute only is not able to deliver equiaxed prior $\beta$-Ti gains, but only decreases the width of columnar grains of additively manufactured Ti-6Al-4V components [7]. Another problem associated with boron additions is the formation of elongated TiB particles at dendrite arm boundaries and/or grain boundaries, which is usually detrimental to the ductility of $\mathrm{Ti}$ alloys [8]. It is worth mentioning that $\mathrm{TiB}_{2}$ is a potent grain refiner for $\beta$ - $\mathrm{Ti}$ in TiAl-based alloys (Al concentration is around 45 at. \%) $[9,10]$. However, $\mathrm{TiB}_{2}$ inoculation is not applicable in common Ti-based alloys, such as $\mathrm{Ti}-6 \mathrm{Al}-4 \mathrm{~V}$, because the $\mathrm{TiB}_{2}$ is not stable in the liquid and will transform to TiB when the Al concentration is lower than 30 at.\% unless an excessive amount of boron (over 8 at.\%) is added [11].

\section{It has been a long-standing challenge to seek potent nucleant particles that can serve as} heterogeneous nucleation sites for prior $\beta$-Ti grains. This is mainly because very few intermetallic phases or even ceramic phases that could act as inoculants, survive in molten titanium which has very high chemical activity at temperatures above $1670^{\circ} \mathrm{C}$. To address 
this challenge, we shifted our focus towards peritectic forming elements because the properitectic phase is thermodynamically stable in liquid titanium. For binary Ti alloys, one of the most common peritectic forming elements is nitrogen, which can form a pro-peritectic phase, TiN, in the Ti-N liquid when the nitrogen content reaches 5 wt. \% [12]. It has been reported in an early US patent that nitrogen can reduce the prior $\beta$-Ti grain size by two to three times when 5 wt. \% nitrogen was added in Ti-6Al-4V [13]. However, such a high concentration is far beyond the acceptance level for engineering applications due to nitrogen embrittlement [14]. In this paper, we present our recent discovery of the refinement of $\beta$-Ti grains at much lower nitrogen content through ZrN inoculation. The grain refining mechanisms of $\mathrm{ZrN}$ inoculation are discussed and the nucleation crystallography is determined.

A typical metastable $\beta$-Ti alloy, Ti-13Mo (wt. \% hereinafter) was selected as a base alloy to retain $\beta$-Ti grains at room temperature. First, $99.7 \%$ pure Ti powder and $99.7 \%$ pure Mo powder were mixed with $99.5 \%$ pure $\mathrm{ZrN}$ powder (from 0 to 8 wt. \%) and blended in a Turbula shaker mixer for half an hour. Another series of samples were made by mixing Ti, Mo powder and $99.5 \%$ pure TiN powder to study the individual effect of nitrogen. The total amount of powder mixture weighs 10 grams. The well blended powder mixture was then compacted into a cylindrical block with the pressure of $100 \mathrm{MPa}$. Cylindrical blocks were arc melted and cooled in a water-chilled copper crucible protected by an argon atmosphere. Each block was melted four times, with the ingot inverted between each re-melting, to enhance the homogeneity of the button ingot. As-cast button ingots were then sliced vertically followed by mechanical polishing. The polished samples were etched by Kroll's 
reagent to reveal the grain boundaries. Energy dispersive spectroscopy (EDS) and electron back-scattered diffraction (EBSD) mapping were conducted at the centre of the vertical cross-section of the button ingots on an FEI Nova NanoSEM at 20 KV. ICP-AES analysis was used to determine the chemical composition of as-cast samples.

The measured alloy composition of the button ingots with different $\mathrm{ZrN}$ additions is shown in Table 1. For brevity, and because the $\mathrm{Zr}$ and $\mathrm{N}$ contents increase equally with $\mathrm{ZrN}$ addition, $\mathrm{ZrN} n(n=2,4,6,8)$ is used as the alloy designation where $n$ represents the weight percentage of $\mathrm{ZrN}$ addition used in this study. The same alloy designation rule also applies to the samples inoculated with TiN. The morphology and size of the $\beta$-Ti grains at different addition levels of $\mathrm{ZrN}$ are shown by $1 \mathrm{~mm} \times 1 \mathrm{~mm}$ EBSD maps in Figure 1. Large columnar grains ( $2 \pm 0.5 \mathrm{~mm}$ long and $0.5 \pm 0.1 \mathrm{~mm}$ wide) were observed in the base alloy. The grain morphology and size are not noticeably different in the ZrN2 and ZrN4 samples. In contrast, fine, equiaxed $\beta$-Ti grains appear in the ZrN6 sample where the average $\beta$-Ti grain size dramatically reduces to $104 \pm 25 \mu \mathrm{m}$. Further addition of $\mathrm{ZrN}$ only reduces the grain size slightly to $80 \pm 15 \mu \mathrm{m}$, however, large primary dendritic nitride particles can be seen inside the $\beta$-Ti grains in the ZrN8 sample (Figure 1e). The difference between Figure $1(a, b, c)$ and $1(d, e)$ implies that a columnar-to-equiaxed transition (CET) and significant grain refinement

Table 1 The chemical composition of the samples in the current study

\begin{tabular}{lcccccc}
\hline Sample description & Designation & $\mathrm{Ti}$ & $\mathrm{Mo}$ & $\mathrm{Zr}$ & $\mathrm{N}$ & $\mathrm{O}$ \\
\hline Ti-13Mo & base alloy & Bal. & 13.3 & --- & 0.03 & 0.14 \\
Ti-13Mo + 2 wt.\% ZrN & ZrN2 & Bal. & 12.9 & 2.00 & 0.14 & 0.19 \\
Ti-13Mo + 4 wt.\% ZrN & ZrN4 & Bal. & 13.4 & 4.11 & 0.26 & 0.26 \\
Ti-13Mo + 6 wt.\% ZrN & ZrN6 & Bal. & 13.4 & 5.84 & 0.40 & 0.32 \\
Ti-13Mo + 8 wt.\% ZrN & ZrN8 & Bal. & 13.5 & 7.78 & 0.52 & 0.34 \\
Ti-13Mo + 4 wt.\% TiN & TiN4 & Bal. & 14.0 & --- & 0.53 & 0.28 \\
\hline
\end{tabular}



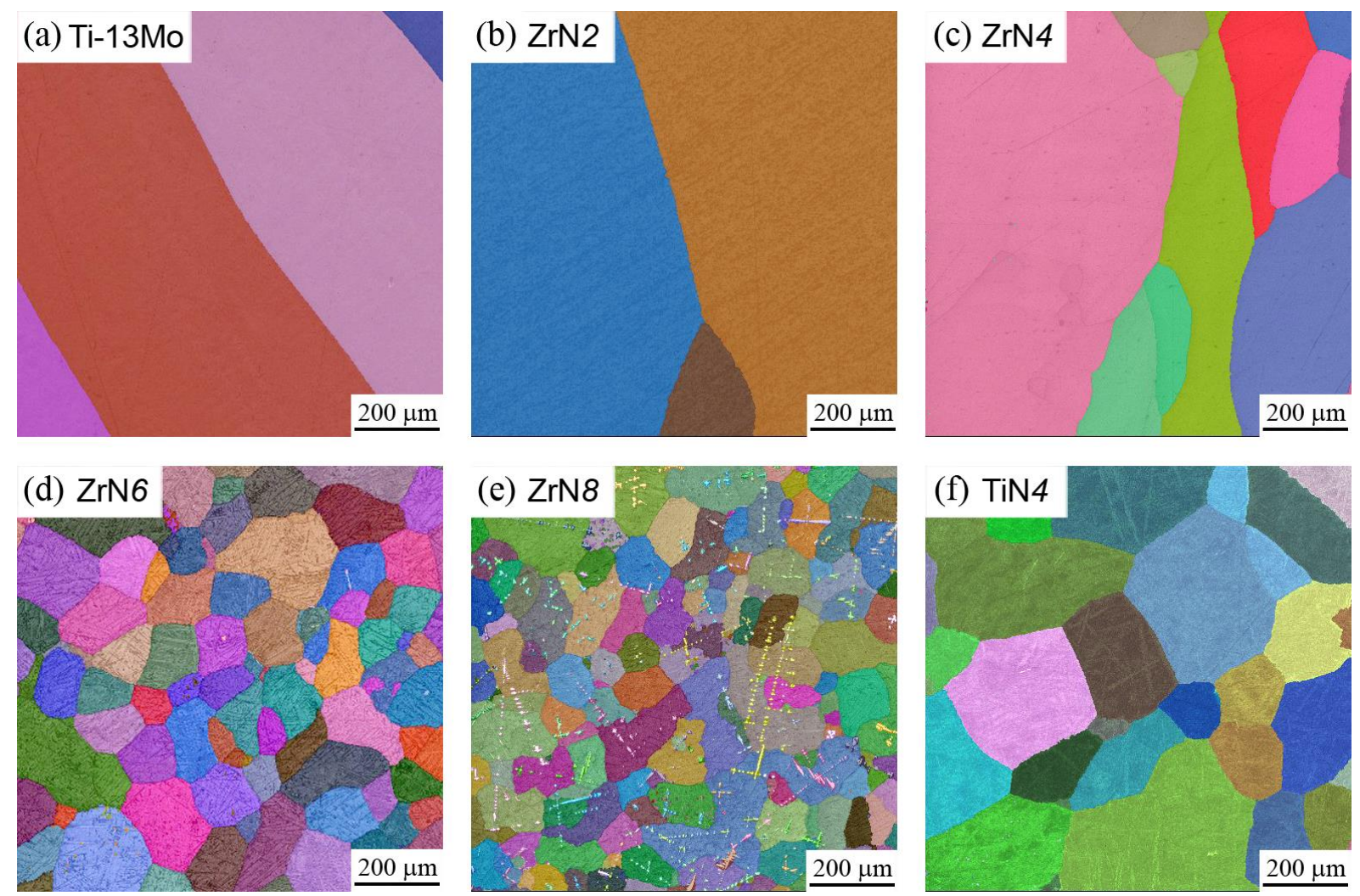

Figure 1 EBSD map of $\beta$-Ti grains in the as-cast button ingot of (a) base alloy, (b) ZrN2, (c) ZrN4, (d) ZrN6, (e) ZrN8 and (f) TiN4.

occurred during solidification when the $\mathrm{ZrN}$ addition reaches the critical level of 6 wt. \% (where the nitrogen content is 0.4 wt. \%). Similar CET and grain refinement were also observed from as-cast Ti-13Mo- $x \mathrm{~N}$ samples when the nitrogen content reaches a critical level of 0.5 wt. \% (TiN4), as shown in Figure 1(f). Compared to the base alloy, the $\beta$-Ti grain size reduced to $200 \pm 36 \mu \mathrm{m}$ when $0.5 \mathrm{wt}$. \% nitrogen was added, but the grain refinement is not as substantial as that in the ZrN6 sample (Figure 1d).

For in-depth understanding of the grain refining mechanisms, local EBSD and EDS mapping were undertaken on the ZrN8 sample which had the best grain refining effect, as shown in 

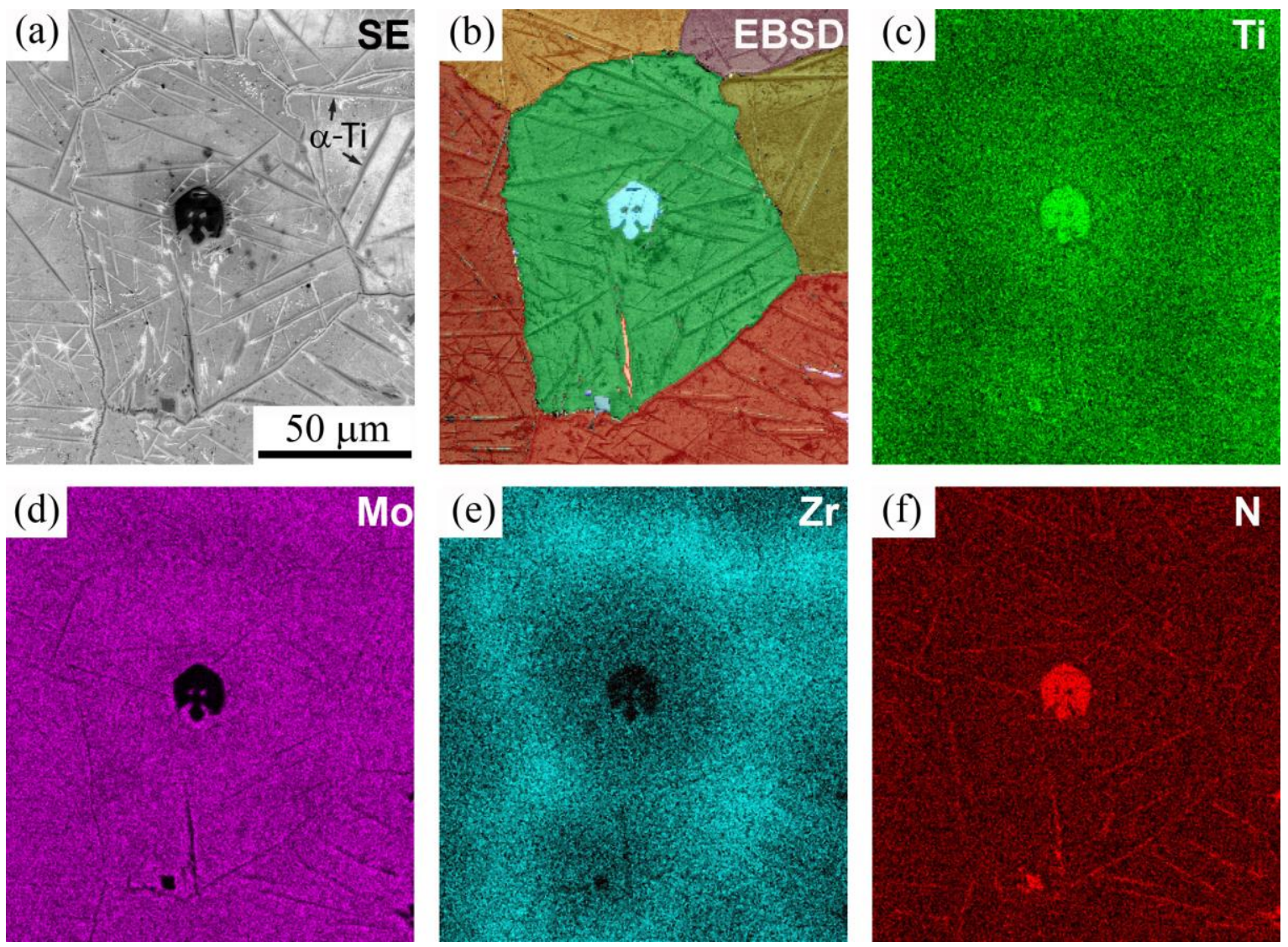

Figure 2 An equiaxed $\beta$-Ti grain in sample ZrN8. (a) secondary electron image. (b) EBSD map. (c-f) EDS map of Ti, Mo, Zr and N.

Figure 2. An equiaxed $\beta$-Ti grain contains a well-defined nucleant particle located at its grain centre. The distribution of four major elements, titanium, molybdenum, zirconium and nitrogen at the same location is shown in Figures 2(c) to 2(f), respectively. High intensities of both nitrogen and titanium were observed inside the nucleant particle whilst the molybdenum and zirconium intensities are very low. This implies that the nucleant particle located at the grain centre is TiN. EBSD analysis shown in Figure 2(b) also confirms this particle is TiN with a face-centred cubic (FCC) crystal structure. Figure 3(a) provides the pole figures of this TiN particle and its surrounding $\beta$-Ti matrix. It can be clearly seen that one of the $\{111\}_{\text {TiN }}$ planes shares the same orientation as one of the $\{110\}_{\beta \text {-Ti }}$ planes (circled in red) 
in the pole figure while one of the $\langle 110\rangle_{\mathrm{TiN}}$ directions parallels one of the $\langle 001\rangle_{\beta-\mathrm{Ti}}$

107 directions (circled in blue). Accordingly, the $\beta$-Ti matrix holds a well-defined Nishiyama-

108 Wassermann (N-W) orientation relationship (OR) with this TiN nucleant particle, which can

109 be expressed as

$110[001]_{\beta-\mathrm{Ti}} / /[\overline{1} 01]_{\mathrm{TiN}},(\overline{1} 10)_{\beta-\mathrm{Ti}} / /(111)_{\mathrm{TiN}}$.

111
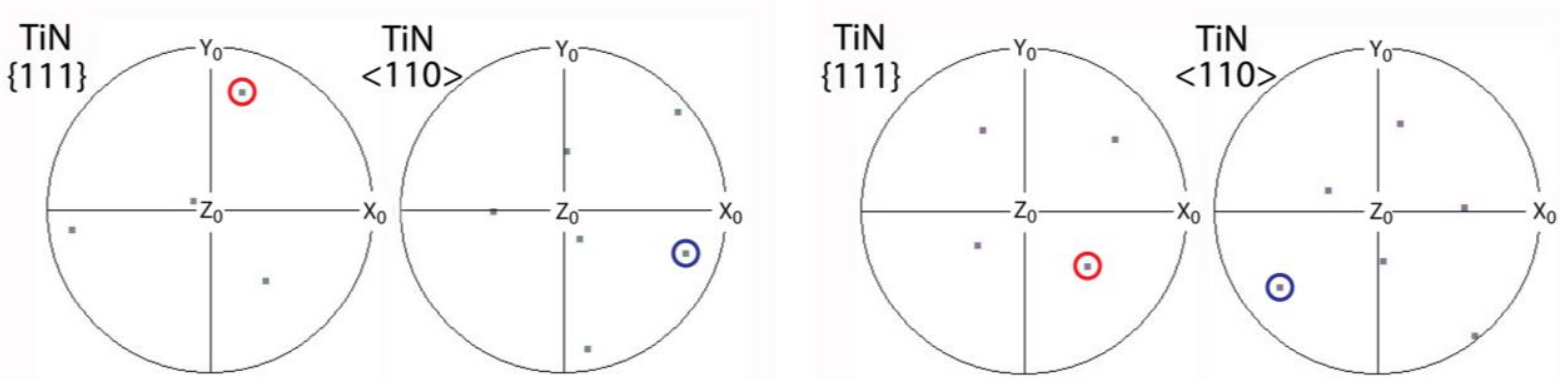

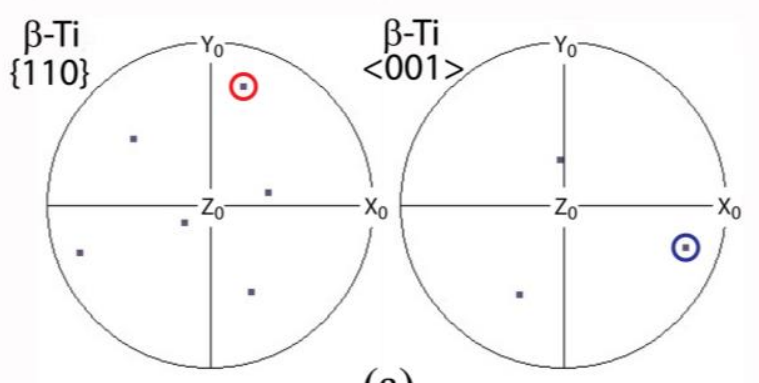

(a)

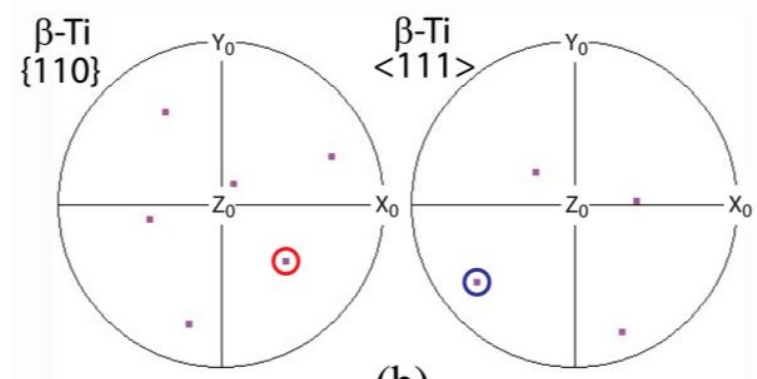

(b)

Figure 3 Pole figures of a TiN nucleant and its surrounding $\beta$-Ti matrix, which presents (a) NW OR and (b) K-S OR.

112 Overall, reproducible ORs were observed from twenty one TiN particles at grain centres

113 after investigating more than fifty $\beta$-Ti grains. Among these twenty one particles, fifteen TiN

114 particles hold the aforementioned N-W OR with their $\beta$-Ti matrix while the second OR was

115 identified from the other six TiN particles. The representative pole figures of the second OR

116 are shown in Figure 3(b). This OR can be expressed as

$117[111]_{\beta-\mathrm{Ti}} / /[\overline{1} 01]_{\mathrm{TiN}},(\overline{1} 10)_{\beta-\mathrm{Ti}} / /(111)_{\mathrm{TiN}}$, 
which corresponds to the Kurdjumov-Sachs (K-S) OR, another common OR between FCC and body centred cubic (BCC) phases.

As an energetically favourable OR is always associated with a local minimum of interfacial energy, the edge-to-edge matching (E2EM) model $[15,16]$ is used to examine the crystallographic matching across the interface between $\mathrm{TiN}$ and $\beta$-Ti. The lattice parameters of TiN and $\beta$-Ti are $0.4225 \mathrm{~nm}$ and $0.3278 \mathrm{~nm}$, respectively, measured by XRD from the ZrN6 sample. There are two pairs of matching directions, $\langle 111\rangle_{\beta-\mathrm{Ti}_{\mathrm{i}}}|<\overline{1} 01\rangle_{\mathrm{TiN}}$ and $\langle 001\rangle_{\beta-\mathrm{Ti}} \mid[\overline{1}$ $01]_{\mathrm{TiN}}$ and two pairs of matching planes, $\{110\}_{\beta-\mathrm{Ti}} \mid\{111\}_{\mathrm{TiN}}$ and $\{\overline{1} 10\}_{\beta-\mathrm{Ti}} \mid\{020\}_{\mathrm{TiN}}$ within the $10 \%$ misfit threshold. After grouping the matching directions and matching planes and applying the $\Delta \mathbf{g}$ parallelism rules [17], two independent ORs are predicted, i.e.

Compared with the measured ORs, the predicted ORs A and B are fully consistent with the KS and N-W ORs, respectively, within the experimental uncertainty.

In addition to the characterization of the nucleant particles, the distribution of solute elements was also studied. As can be seen from the EDS maps in Figures 2(e) and 2(d), the majority of zirconium segregates along the $\beta$-Ti grain boundaries whilst molybdenum is

137 depleted from the grain boundaries. This indicates that substantial partitioning of both zirconium and molybdenum solute occurs across the solid/liquid interface during solidification. Solute zirconium and molybdenum partition in an opposite way because $\mathrm{Ti}-\mathrm{Zr}$ 
and Ti-Mo systems have an opposite sign for the gradient of the liquidus line on the Ti-rich

141 side.

Based on the above experimental studies, the mechanisms of CET and grain refinement of $\beta$ -

Ti grains through $\mathrm{ZrN}$ inoculation can be attributed to the synergistic effect of in-situ formed

TiN particles and enhanced solute segregation, as graphically illustrated in Figure 4 . For the

Ti-13Mo base alloy, the columnar grains nucleated from the bottom of the water-chilled copper crucible and grew in the opposite direction to the heat extraction direction, i.e. from the edge to the thermal centre of the melt pool. Because of the lack of potent nucleant particles in the melt pool, the critical undercooling required for nucleation, $\Delta T_{n}$, is too large to be met by the constitutional supercooling, $\Delta T_{c s}$, delivered by solute molybdenum. As a result, no equiaxed grains formed and large columnar grains grew across the whole vertical cross-section (Figure 4a).

When the alloy melt was inoculated with ZrN, it appears that the $\mathrm{ZrN}$ particles quickly decomposed when the powder compact was completely melted, as no ZrN phase was identified in the as-cast button ingot, i.e.

where $[\mathrm{Zr}]_{\text {liquid }}$ and $[\mathrm{N}]_{\text {liquid }}$ represent the solute zirconium and nitrogen released into the liquid titanium from the decomposition of ZrN. However, there is still no potent nucleant particle available in the ZrN2 and ZrN4 samples, and hence columnar grains still dominate 
(a) Ti-13Mo

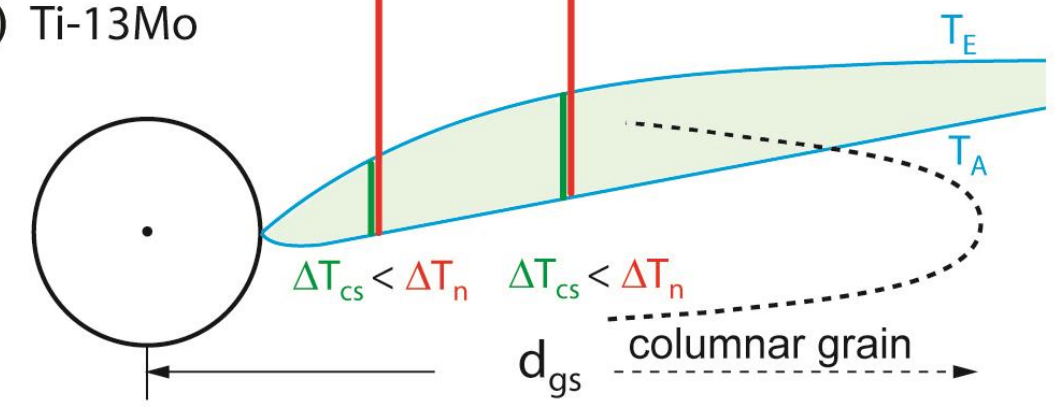

(b) $\mathrm{Ti}-13 \mathrm{Mo}-0.5 \mathrm{~N}$

$$
\Delta \mathrm{T}_{\mathrm{cs}}=\Delta \mathrm{T}_{\mathrm{n}}
$$

$\mathrm{T}_{\mathrm{E}}$

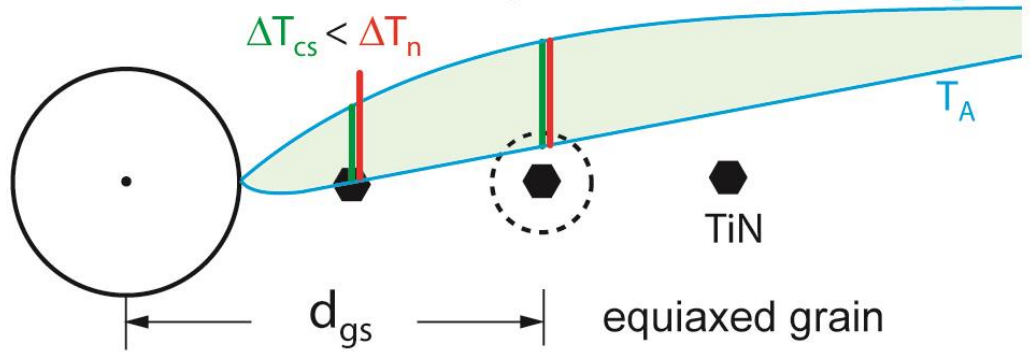

(c) $\mathrm{Ti}-13 \mathrm{Mo}-6 \mathrm{Zr}-0.4 \mathrm{~N}$

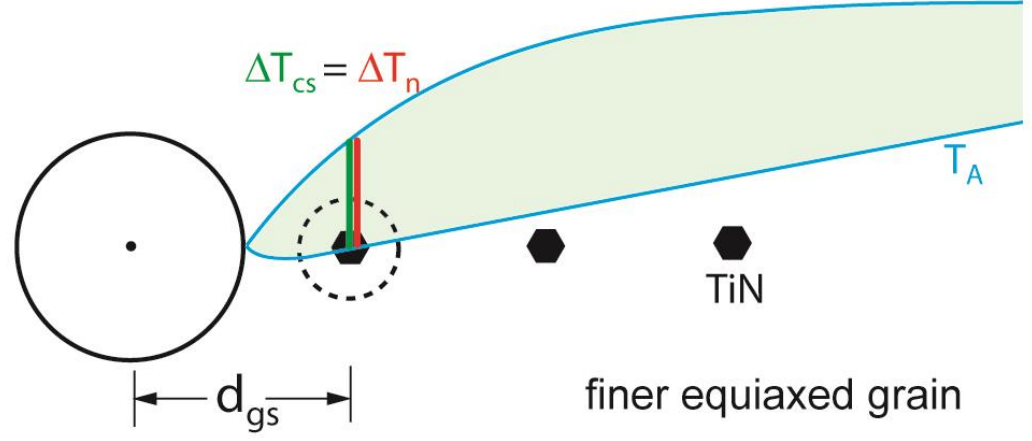

Figure 4 Graphic illustration of the mechanism of columnar dendrite formation in (a) base alloy, and mechanisms of CET and grain refinement in (b) Ti-13Mo-0.5N alloy (TiN4) and (c) Ti-13-Mo-6Zr-0.4N alloy (ZrN6). The constitutionally supercooled zone is shaded in light blue. The amount of $\Delta \mathrm{T}_{\mathrm{cs}}$ and $\Delta \mathrm{T}_{\mathrm{n}}$ is qualitatively represented by the length of green bar and red bar, respectively. $T_{E}$ and $T_{A}$ represent the equilibrium liquidus temperature and actual temperature profile, respectively. The average grain size is denoted by $d_{g s}$. 
the microstructure. When the addition of $\mathrm{ZrN}$ induced $0.4 \mathrm{wt}$. \% nitrogen (Sample ZrN6) or

164 the addition of TiN induced 0.5 wt. \% nitrogen (Sample TiN4) in the liquid, primary TiN 165 particles start to form from the liquid prior to the formation of $\beta$-Ti, i.e.

These TiN particles provide potent heterogeneous nucleation sites for $\beta$-Ti with much smaller $\Delta \mathrm{T}_{\mathrm{n}}$ required for nucleation due to good crystallographic matching with $\beta$-Ti (Figures $4 b$ and $4 c)$.

When the first $\beta$-Ti dendrite forms in the liquid, the partitioning of both solute zirconium and molybdenum contribute to the formation of a constitutionally supercooled zone formed ahead of the solid-liquid interface. Once the development of $\Delta T_{\text {cs }}$ fulfils the $\Delta T_{n}$ of a TiN particle located in the constitutionally supercooled zone, this TiN particle will be activated as a nucleation site and a new equiaxed $\beta$-Ti dendrite is formed around it (Figure 4c). The formation of new equiaxed $\beta$-Ti dendrites effectively stops columnar grain growth through grain impingement. According to the Interdependence theory $[18,19]$, when the first equiaxed $\beta$-Ti dendrite forms in the liquid, the constitutionally supercooled zone will continue to trigger successive heterogeneous nucleation events on the available potent TiN particles towards the thermal centre of the cooling melt pool. As a result, the microstructure is dominated by fine, equiaxed $\beta$-Ti dendrites when solidification is complete, leaving excess zirconium and depleted molybdenum along the dendritic arm boundaries and grain boundaries and active TiN nucleant particles at the centre of $\beta$-Ti grains. 
187 The growth restriction factor (or Q-value) of individual solute elements is a common parameter to evaluate their capability to developing constitutional supercooling [19]. Solute molybdenum provides fairly high constitutional supercooling based on its Q-value of $85 \mathrm{~K}$ at 13 wt. \% [20]. This explains why the CET and grain refinement can also be observed in

Sample TiN4 when only 0.5 wt. \% nitrogen was added into the Ti-13Mo base alloy (Figure

4b). The segregation of solute zirconium offers additional constitutional supercooling so that

$\Delta T_{n}$ of the available TiN particles can be reached at a much shorter distance ahead of the

first $\beta$-Ti dendrite. This explains why the grain size of the ZrN6 sample (Figure 4c) is half as

small as the TiN4 sample (Figure 4b). It is also noteworthy that the Q-value of solute

zirconium itself is almost negligible (e.g. less than $2 \mathrm{~K}$ even at $6 \mathrm{wt} . \%$ ) [21], which appears

contradictory to the strong partition of solute zirconium observed in the current study. This

implies that the actual constitutional supercooling in a multicomponent alloy cannot be

directly evaluated by simple addition of the Q-values from individual solute elements

deduced from a binary phase diagram. A more comprehensive investigation should be

undertaken to establish a more accurate phase diagram in the specific multicomponent Tibased alloy under consideration, so that the Q-value can be quantified rigorously by the initial rate of development of constitutional supercooling [22]. alloy and it can be further reduced to $0.4 \mathrm{wt} . \%$ if $\mathrm{ZrN}$ is inoculated. In contrast to binary Ti-N alloys where the minimal concentration of nitrogen to form pro-peritectic TiN phase is 5 
wt. \%, much less nitrogen is needed for refining the $\beta$-Ti grains in a $\beta$-Ti alloy. In addition, nitrogen embrittlement might not be an issue in $\beta$-Ti alloys. This is because nitrogen is an $\alpha-$

211 Ti stabilizer and stimulates $\alpha$-Ti precipitation from the $\beta$-Ti matrix even at a fairly high cooling rate (in the order of $10^{2} \mathrm{~K} / \mathrm{s}$ ) during the arc melting process (Figure 2a). At room temperature, the majority of nitrogen is apt to remain in the $\alpha$ precipitates, rather than in the $\beta$-Ti matrix (Figure 2f). Figure 5 shows the effect of $\mathrm{ZrN}$ addition on the hardness of Ti13Mo base alloy. The hardness increases from $305 \mathrm{HV}$ to $411 \mathrm{HV}$ after 6 wt.\% ZrN was added into Ti-13Mo. The remarkable hardness increment is attributed to the combination of

(a) Ti-13Mo

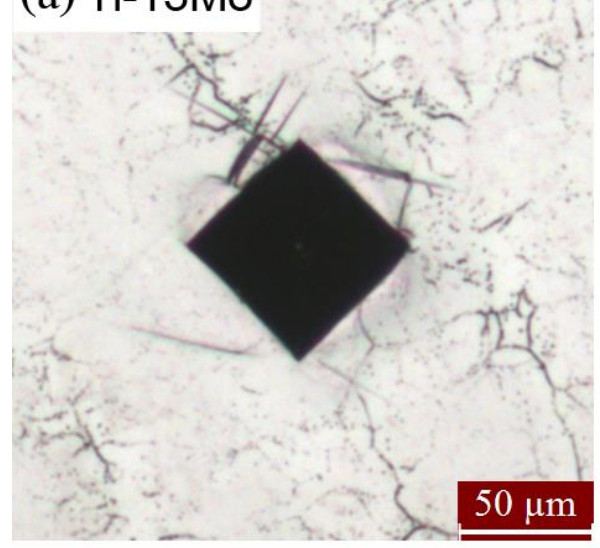

(c) 450

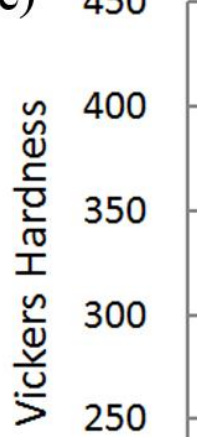

(b) ZrN6

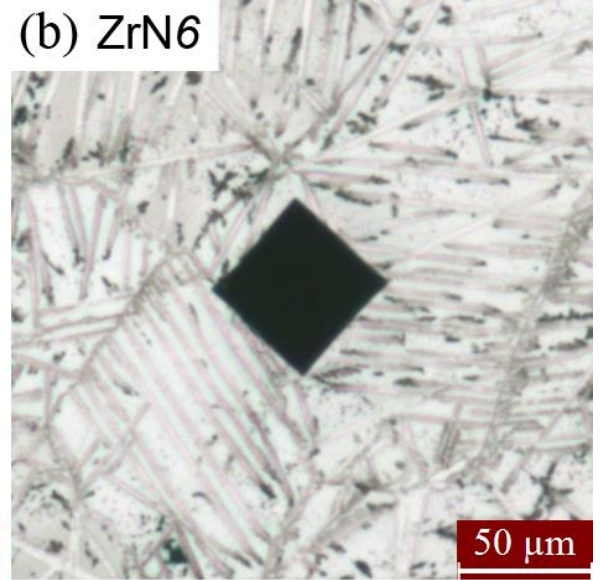
200

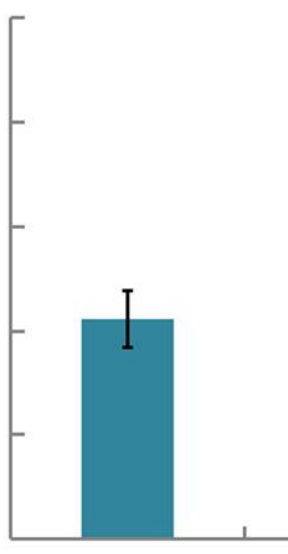

Ti-13Mo

$\mathrm{ZrN2}$

ZrN6

Figure 5 Vickers indentation on as-cast (a) Ti-13Mo and (b) ZrN6 samples after applying $1 \mathrm{~kg}$ load for 10 seconds. (c) Effect of ZrN addition on Vickers hardness of Ti-13Mo alloy. 
additional solid solution strengthening from solute $\mathrm{Zr}$, precipitation strengthening from $\alpha$ precipitates as well as grain boundary strengthening from grain refinement. It should also be noted that no cracks was observed around the corners of indentation in any $\mathrm{ZrN}$ inoculated samples during Vickers hardness test, which indicates a reasonable ductility. Therefore, smart manipulation of nitrogen and/or zirconium within a $\beta$-Ti alloy could offer a costeffective way to develop new high-strength $\beta$-Ti alloys.

In summary, coarse columnar $\beta$-Ti grains can be converted to fine equiaxed grains in as-cast, Ti-Mo based $\beta$-Ti alloys when a brand new inoculant, $\mathrm{ZrN}$ is added into the molten Ti at a nitrogen level as low as $0.4 \mathrm{wt} . \%$. The substantial grain refinement is attributed to the heterogeneous nucleation of $\beta$-Ti around in-situ formed TiN particles and enhanced constitutional supercooling delivered by solute zirconium. In addition to the grain refinement, addition of $\mathrm{ZrN}$ also induces remarkable hardness increment due to the extra solid solution strengthening from solute zirconium and precipitation strengthening from nitrogen-rich a precipitates.

\section{Acknowledgement}

The authors wish to acknowledge the Australian Research Council (ARC) for the financial support (DP160100560). The authors are indebted to Dr Daniel East and Dr Arvind Prasad for the sample preparation of $\mathrm{ZrN}$ inoculated Ti button ingots. The authors would also like to acknowledge the technical assistance of the RMIT Microscope and Microanalysis Facility, RMIT University. 
[1] A. Lasalmonie and J.L. Strudel: J. Mater. Sci., 1986, vol. 21, pp. 1837-52.

[2] Q. Zhang, J. Chen, H. Tan, X. Lin and W.D. Huang: J. Alloys Compds., 2016, vol. 666, pp. 380-86.

[3] T. Wang, Y.Y. Zhu, S.Q. Zhang, H.B. Tang and H.M. Wang: J. Alloys Compds., 2015, vol. 632, pp. 505-13.

[4] J.H. Martin, B.D. Yahata, J.M. Hundley, J.A. Mayer, T.A. Schaedler and T.M. Pollock: Nature, 2017, vol. 549, pp. 365-69.

[5] M.J. Bermingham, S.D. McDonald, K. Nogita, D.H. StJohn and M.S. Dargusch: Scripta Mater., 2008, vol. 59, pp. 538-41.

[6] S. Tamirisakandala, R.B. Bhat, J.S. Tiley and D.B. Miracle: Scripta Mater., 2005, vol. 53, pp. 1421-26.

[7] M.J. Bermingham, D. Kent, H. Zhan, S.D. McDonald, D.H. StJohn and M.S. Dargusch: Acta Mater., 2015, vol. 91, pp. 289-303.

[8] R. Sarkar, P. Ghosal, K. Muraleedharan, T.K. Nandy and K.K. Ray: Mater. Sci. Eng. A, 2011, vol. 528, pp. 4819-29.

[9] J.D. Bryant, L. Christodoulou and J.R. Maisano: Scripta Mater., 1990, vol. 24, pp. 33-38.

[10] D. Gosslar, R. Günther, U. Hecht, C. Hartig and R. Bormann: Acta Mater., 2010, vol. 58, pp. 6744-51.

[11] V.T. Witusiewicz, A.A. Bondar, U. Hecht, J. Zollinger, L.V. Artyukh and T.Ya. Velikanova: J. Alloys Compds., 2009, vol. 474, pp. 86-104.

[12] H. A. Wriedt and J. L. Murray: Binary Alloy Phase Diagrams, vol. 3, ASM, Metals Park, OH, 1986, pp. 2705.

[13] F.A. Crossley and P.A. Calif: Grain refinement of titanium alloys, United States Patent No. 4420460, 1983.

[14] H. R. Ogden and R. L. Jaffee: TML Report, No. 20, Battelle Memorial Institute, 1955, pp. 20.

[15] M.-X. Zhang, P.M. Kelly, M.A. Easton and J.A. Taylor: Acta Mater., 2005, vol. 53, pp. 1427-38.

[16] D. Qiu, M.-X. Zhang, J.A. Taylor and P.M. Kelly: Acta Mater., 2009, vol. 57, pp. 3052-59.

[17] W.-Z. Zhang and G.C. Weatherly: Prog. Mater. Sci., 2005, vol. 50, pp. 181-292.

[18] D.H. StJohn, Q. Ma, M.A. Easton and P. Cao: Acta Mater., 2011, vol. 59, pp. 4907-4921.

[19] D.H. StJohn, A. Prasad, M.A. Easton and M. Qian: Metall. Mater. Trans. A, 2015, vol. 46, pp. 4868-85.

[20] M.J. Bermingham, S.D. McDonald, M.S. Dargusch and D.H. StJohn: J. Mater. Res., 2008, vol. 23, pp. 97-104.

[21] M.J. Bermingham, S.D. McDonald, D.H. StJohn and M.S. Dargusch: J. Alloys Compds., 2009, vol. 481, pp. L20-23.

[22] R. Schmid-Fetzer and A. Kozlov: Acta Mater., 2011, vol. 59, pp. 6133-44. 
281

282

283

284

285

286

287

288

289

290

291

292

293

294

295

296

297

298

299

300

301

302

Table Title

Table 1The chemical composition of the samples in the current study.

\section{Figure Caption}

Figure 1 EBSD map of $\beta$-Ti grains in the as-cast button ingot of (a) base alloy, (b) ZrN2, (c) ZrN4, (d) ZrN6, (e) ZrN8 and (f) TiN4.

Figure 2 An equiaxed $\beta$-Ti grain in sample ZrN8. (a) secondary electron image. (b) EBSD map. (c-f) EDS map of Ti, Mo, Zr and N.

Figure 3 Pole figures of a TiN nucleant and its surrounding $\beta$-Ti matrix, which presents (a) N-W OR and (b) K-S OR.

Figure 4 Graphic illustration of the mechanism of columnar dendrite formation in (a) base alloy, and mechanisms of CET and grain refinement in (b) Ti-13Mo-0.5N alloy (TiN4) and (c) Ti-13-Mo-6Zr-0.4N alloy (ZrN6). The constitutionally supercooled zone is shaded in light blue. The amount of $\Delta T_{\text {cs }}$ and $\Delta T_{n}$ is qualitatively represented by the length of green bar and red bar, respectively. $T_{E}$ and $T_{A}$ represent the equilibrium liquidus temperature and actual temperature profile, respectively. The average grain size is denoted by $d_{\mathrm{gs}}$.

Figure 5 Vickers indentation on as-cast (a) Ti-13Mo and (b) ZrN6 samples after applying 1 kg load for 10 seconds. (c) Effect of ZrN addition on Vickers hardness of Ti-13Mo alloy. 
Table 1 The chemical composition of the samples in the current study

\begin{tabular}{lllllll}
\hline Sample description & Designation & $\mathrm{Ti}$ & $\mathrm{Mo}$ & $\mathrm{Zr}$ & $\mathrm{N}$ & $\mathrm{O}$ \\
\hline Ti-13Mo & base alloy & Bal. & 13.3 & --- & 0.03 & 0.14 \\
Ti-13Mo + 2 wt.\% ZrN & ZrN2 & Bal. & 12.9 & 2.00 & 0.14 & 0.19 \\
Ti-13Mo + 4 wt.\% ZrN & ZrN4 & Bal. & 13.4 & 4.11 & 0.26 & 0.26 \\
Ti-13Mo + 6 wt.\% ZrN & ZrN6 & Bal. & 13.4 & 5.84 & 0.40 & 0.32 \\
Ti-13Mo + 8 wt.\% ZrN & ZrN8 & Bal. & 13.5 & 7.78 & 0.52 & 0.34 \\
Ti-13Mo + 4 wt.\% TiN & TiN4 & Bal. & 14.0 & --- & 0.53 & 0.28 \\
\hline
\end{tabular}


(a) Ti-13Mo

\section{(d) ZrN6}

(b) ZrN2

$200 \mu \mathrm{m}$

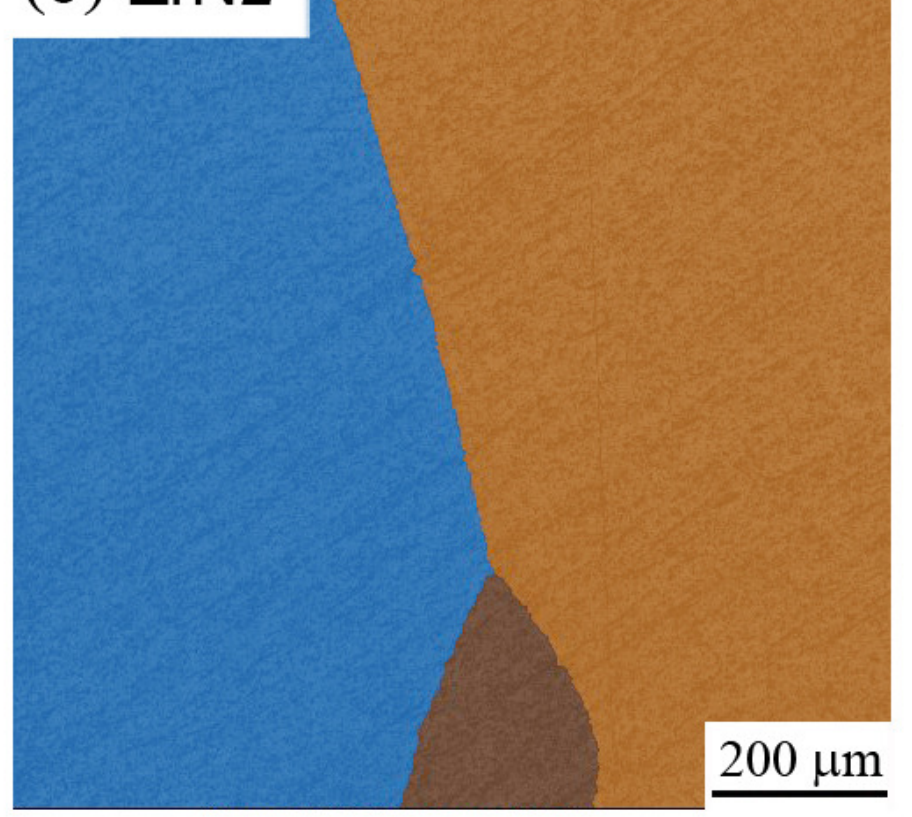

(c) $\mathrm{ZrN} 4$

$200 \mu \mathrm{m}$

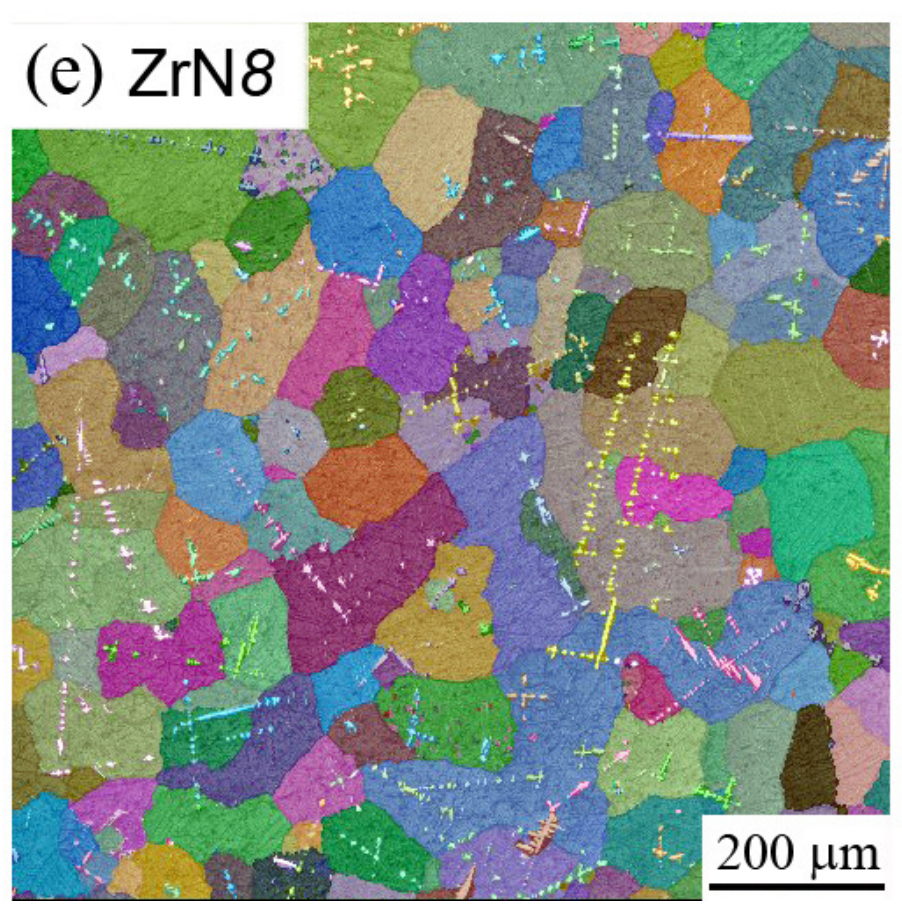

(f) TiN4

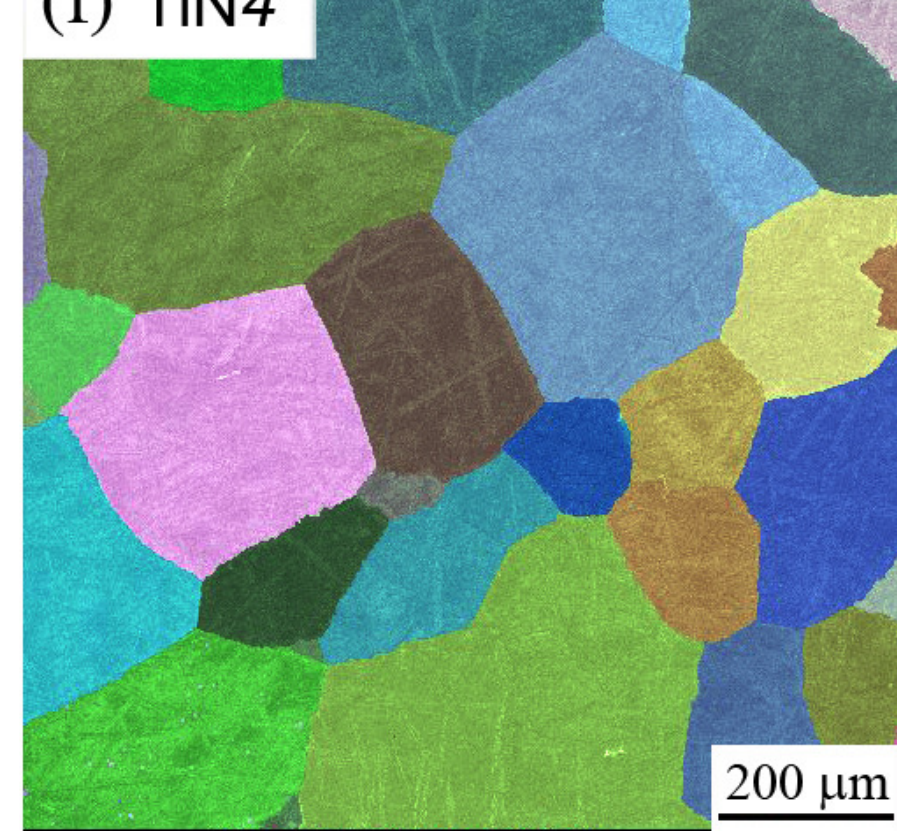



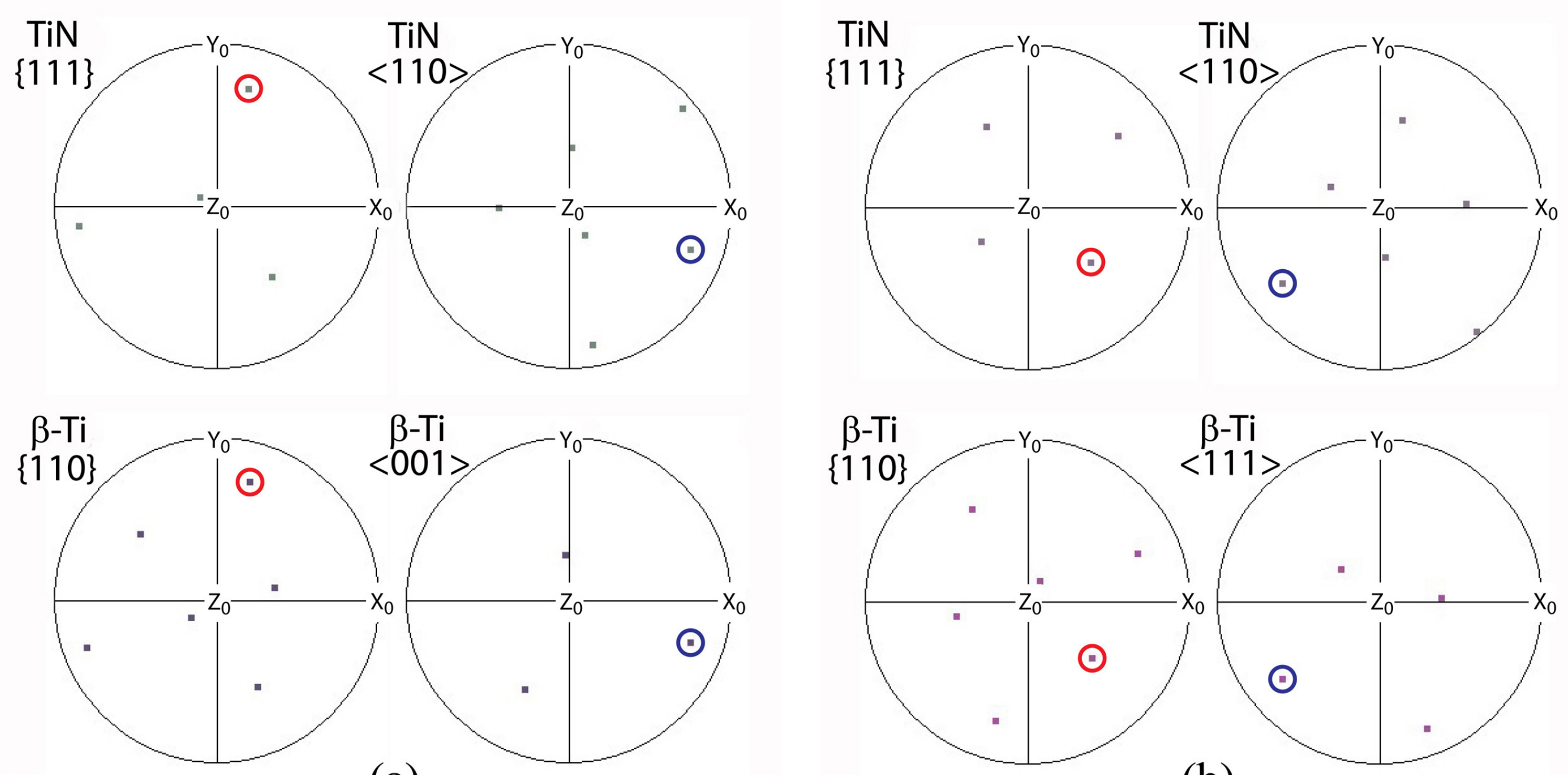

(a)

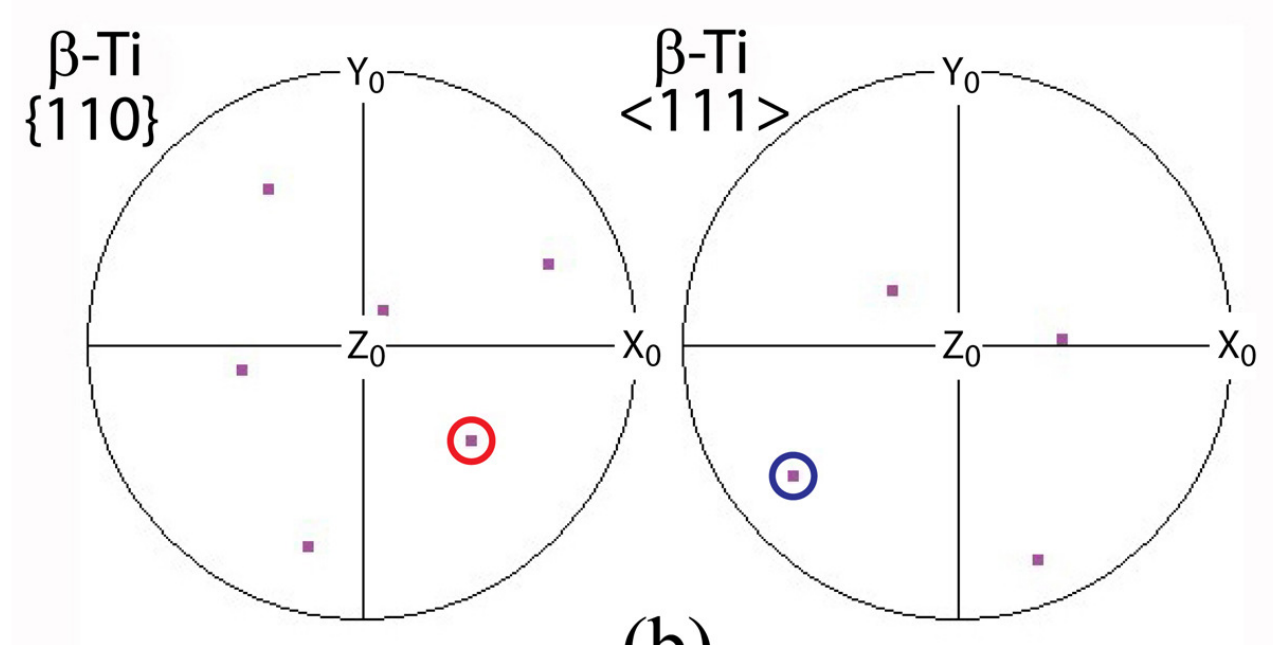

(b) 
(a) $\mathrm{Ti}-13 \mathrm{Mo}$

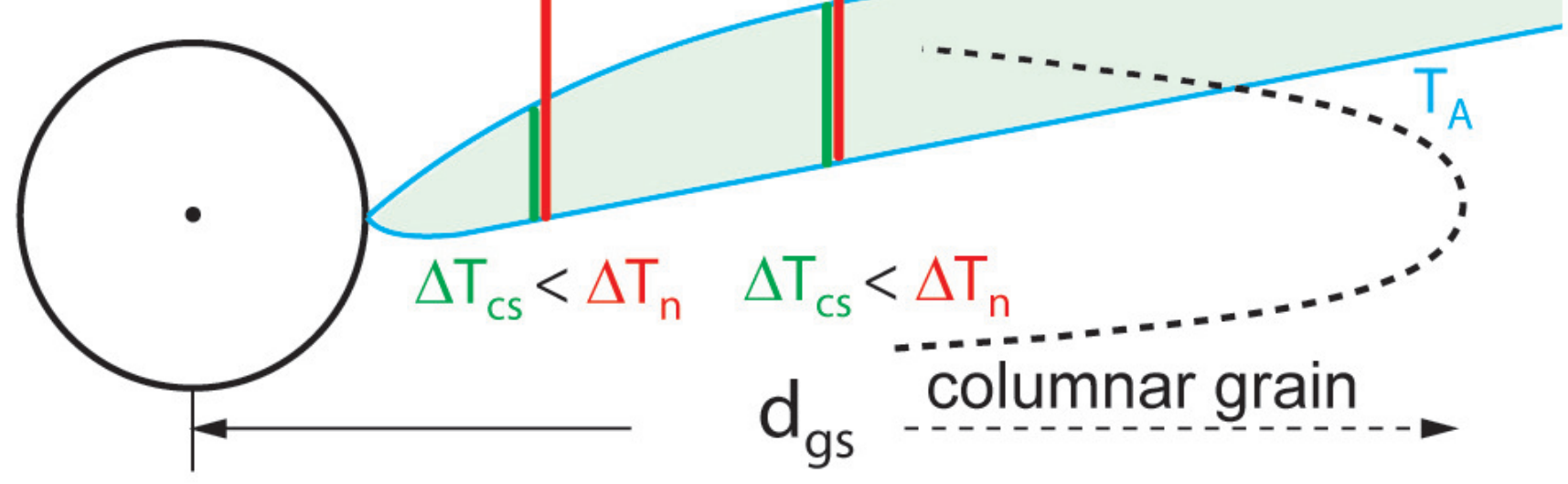

(b) $T \mathrm{i}-13 \mathrm{Mo}-0.5 \mathrm{~N}$

$\Delta \mathrm{T}_{\mathrm{cs}}=\Delta \mathrm{T}_{\mathrm{n}}$

$\mathrm{T}_{\mathrm{E}}$

$\Delta \mathrm{T}_{\mathrm{cs}}<\Delta \mathrm{T}_{\mathrm{n}}$

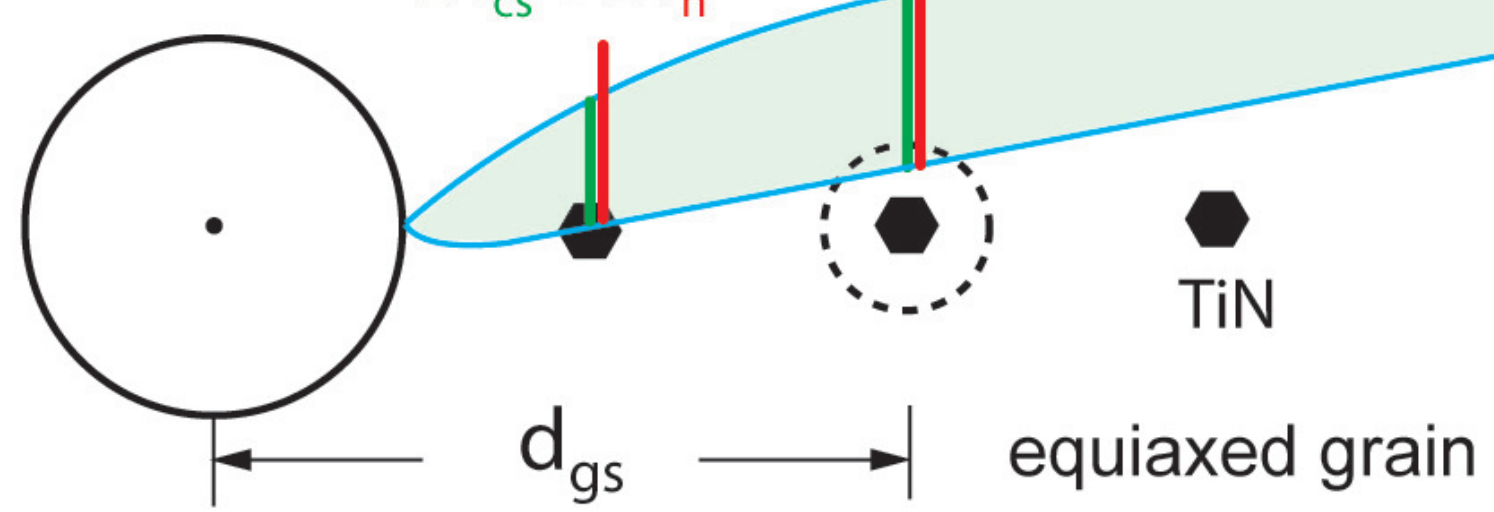

(c) $\mathrm{Ti}-13 \mathrm{Mo}-6 \mathrm{Zr}-0.4 \mathrm{~N}$

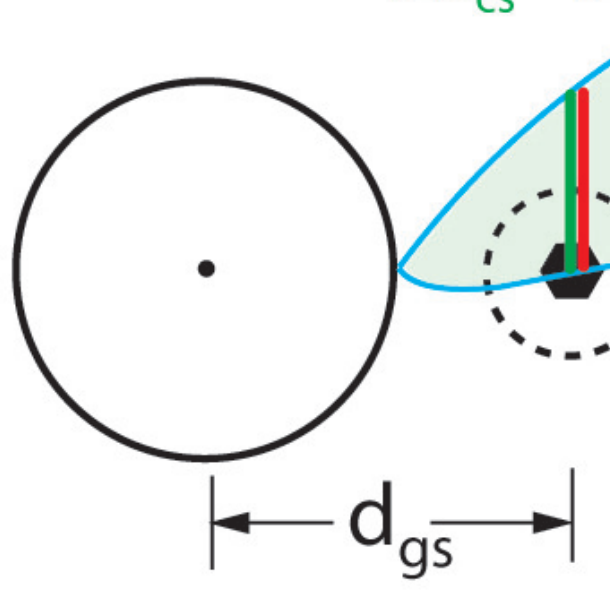

$\mathrm{T}_{\mathrm{A}}$

finer equiaxed grain 
(b) ZrN6 $11,4=$
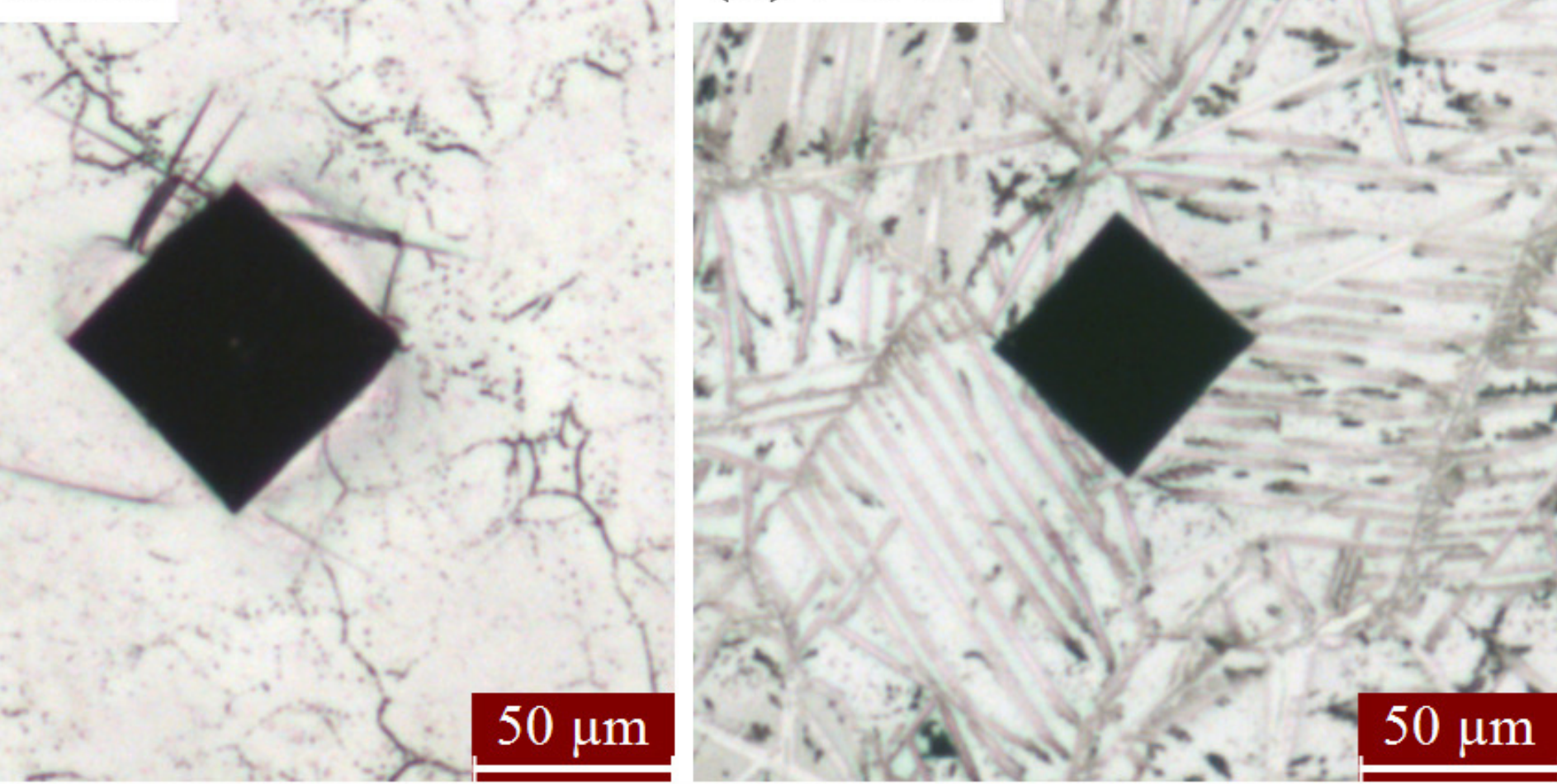

(c) 450

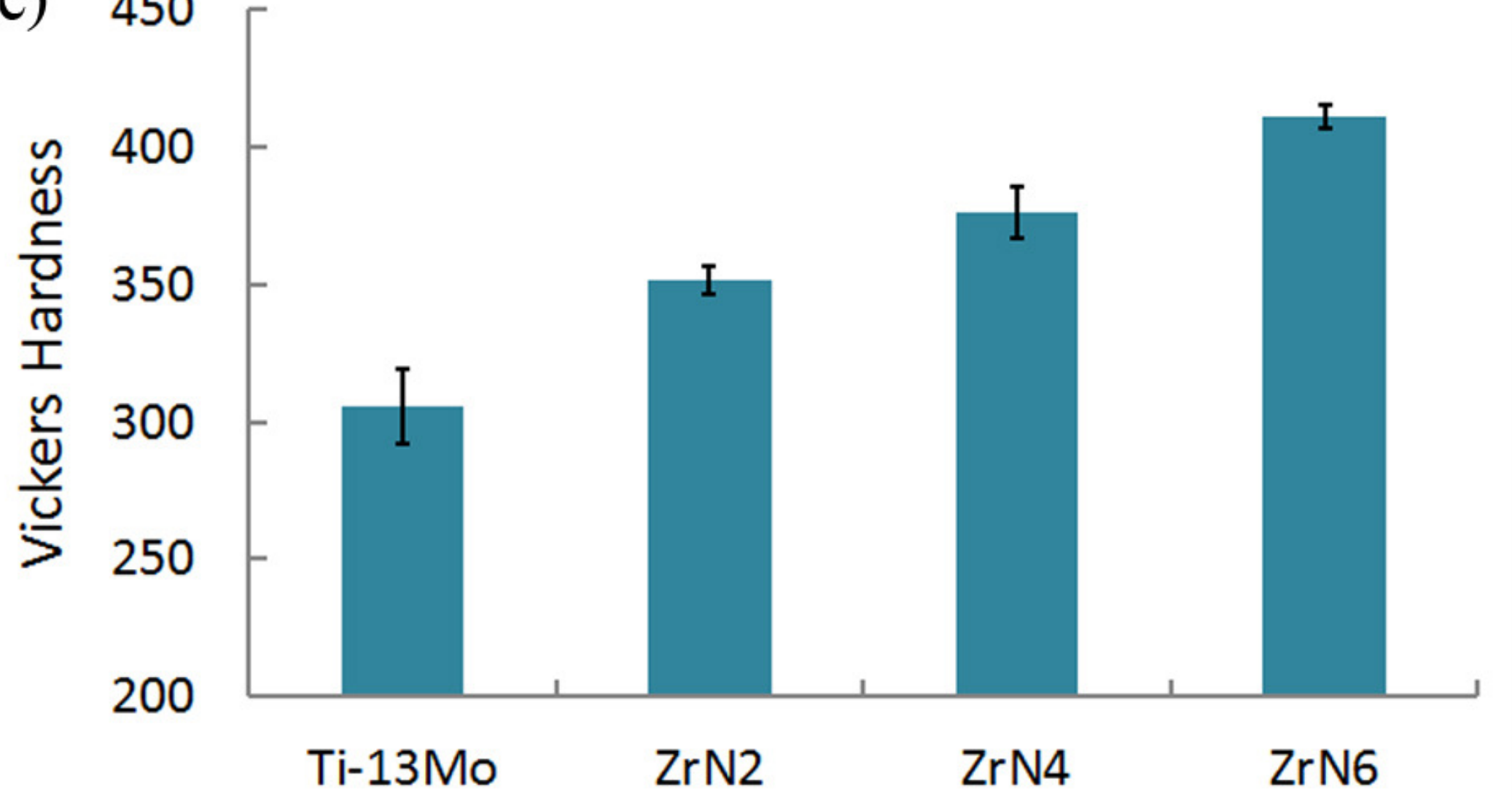

